\title{
Contemporary rehabilitation at cancer centres
}

\author{
Katarzyna Hojan \\ Department of Rehabilitation, the Greater Poland Cancer Centre, Poznań, Poland
}

\begin{abstract}
Aim. Cancer rehabilitation is an important, but often underutilized treatment in the comprehensive care of the cancer patient. The lack of appropriate referral by physicians unfamiliar with the concept of rehabilitation was identified as primary barriers to optimal delivery of rehabilitation care. Therefore, the aim of this paper is to describe the current situation in the world of science of cancer rehabilitation and to describe availability of professional resources in the selected countries in the world.

Material and methods. This paper is a review article to describe rehabilitation models in cancer services for patients in selected cases in the world.

Results. Rehabilitation should be applied in various settings, depending on the level of disability, extent of disease, medical acuity level of the patient, and available services. However, the rehabilitation systems in the world differ depending on the various social security and health-care systems, but they are largely based on a similar, multidimensional and multidisciplinary understanding of cancer rehabilitation.

Conclusions. On the basis of description of the bio-psycho-social models, it can be seen that rehabilitation must be an integral and continuous part of all cancer care. There is strong evidence that rehabilitation is a welltolerated and safe adjunct therapy that can mitigate several common treatment-related side effects among cancer patients.
\end{abstract}

Keywords: oncology, rehabilitation medicine, physical activity, physiotherapy, occupational therapy.

\section{Introduction}

The concept of rehabilitation in cancer care is part of the new situation characterized by a stable rise in incidence of cancer overall in most countries, concurring with a rise in the number of cancer survivors [1]. The lack of identification of patient problems and of appropriate referral by physicians unfamiliar with the concept of rehabilitation were identified as primary barriers to optimal delivery of rehabilitation care.

The World Health Organization (WHO) has defined rehabilitation as "the use of all means aimed at reducing the impact of disabling and handicapping conditions and at enabling people with disabilities to achieve optimal social integration" [2]. The Model of Functional Health as established in the WHO's International Classification of Functioning, Disability and Health (ICF) is considered to provide a theoretical framework of rehabilitation [3, 4]. The ICF complements the International Classification of Diseases (ICD) and provides a conclusive conceptual framework which incorporates the biological, as well as the individual and the social aspects of health conditions $[5,6]$.

Based on the bio-psycho-social model of the WHO and a holistic approach of rehabilitation, the cancer rehabilitation comprises multidisciplinary efforts including medical, psychological and physiotherapeutic treatment, as well as occupational therapy and functional therapy, depending on the patient's functional status. Recommendations have been made for finding better methods for identifying and managing the broader effects of cancer and its treatment and for integrating a more holistic interdisciplinary approach during and after the treatment of patients with cancer. The role of physical therapy is well established in some areas (e.g., post-mastectomy exercises, lymphedema management), and it is exciting to see the programmes that are emerging across the countries and the breadth of involvement of exercises discipline across cancer types and through the continuum of cancer care. 


\section{Aim}

The aims of this paper are to provide a description of the current situation in cancer rehabilitation, to give an overview of the state of science of cancer rehabilitation and to describe availability of professional resources within the general health-care systems in selected countries in the world.

\section{Material and methods}

This paper is a review article to describe professional resources and rehabilitation models in cancer services for patients in selected countries of the world. This article presents rehabilitation programms in the United States, Canada, and Germany as well as an overview of ongoing studies.

\section{Prior to treatment}

The limited available data suggest that short-term, presurgical exercise training is feasible, well-tolerated, and potentially associated with significant improvements in aerobic capacity $[6,7]$.

\section{During cancer therapy}

The vast majority of studies were conducted in patients with early stage disease, predominantly women with breast cancer, receiving conventional cytotoxic therapies such as chemotherapy, radiotherapy, or androgen deprivation therapy (in the case of prostate cancer patients). Results of these studies indicated that supervised exercise training following traditional guidelines [i.e. aerobic training ( $3 \mathrm{~d} / \mathrm{wk}$, for $30-45$ min per session, at $50-75 \%$ of age-predicted heart rate maximum or baseline aerobic capacity for 12-15 weeks) or resistance training ( $3 \mathrm{~d} / \mathrm{wk}, 10$ exercises, at $60-70 \%$ of one-repetition maximum)] was associated with improvements in terms of aerobic capacity, upper and lower extremity muscle strength, functional $\mathrm{QOL}$, and several other psychological outcomes. Overall, the current literature base indicates that supervised exercise therapy is safe and feasible and associated with significant improvements in selected outcomes in patients with early-stage disease receiving conventional cytotoxic adjuvant therapy $[1,6,7,8]$.

\section{Past adjuvant therapy}

Speck et al. [9] reported that $60 \%$ of all studies in cancer rehabilitation were conducted in the post-adjuvant therapy setting. Results of the presented studies indicated that supervised exercise training following traditional guidelines was associated with improvements in measures of aerobic capacity, upper and lower extremity muscle strength, overall QOL, fatigue, and several other psychological outcomes (e.g. mood disturbance) [5-8].

\section{Discussion}

Rehabilitation needs vary greatly across tumour groups and individuals, as the medical effects depend on both the cancer type and the treatment regimen. Cancer can cause multiple impairments and the bio-psycho-social model as a core concept of modern definitions of rehabilitation supports the interdisciplinary team approach to cancer rehabilitation (Table 1). Depending on the cancer disease, patients may suffer from various functional symptoms such as, loss of motor control, cognitive and speech problems, swallowing problems, and sensory loss. Thus, evaluation studies related to cancer rehabilitation cover a wide variety of interventions and programs, ranging from specific treatments such as urinary incontinence training for prostate cancer patients to multidimensional rehabilitation programs covering several interventions from physical exercise to relaxation training and psycho-educational interventions [8, 10]. In general, the existent body of research indicates that rehabilitative interventions reduce symptom distress in cancer patients and increase quality of life, functioning and general well-being. However, the evidence levels for rehabilitative interventions range from good (e.g. for exercises or relaxation training and psychosocial counseling) to low (lymph drainage and art therapy). Multiple studies of cancer patients receiving interdisciplinary rehabilitation in inpatient settings have shown functional gains equal to or better than in control patients, who exhibited similar impairments without having received a cancer diagnosis [10]. Other studies of exercise interventions for cancer patients, both during and after treatments, have demonstrated positive outcomes in several realms, and not just increased exercise tolerance. DePompolo [11] described successful rehabilitation outcomes from an interdisciplinary inpatient consultation team. Hospice based rehabilitation interventions and outcomes have also been documented [12]. An acute inpatient cancer rehabilitation unit within a comprehensive cancer center, using a comprehensive interdisciplinary team, may be most appropriate. Patients referred for inpatient rehabilitation tend to be those with multiple impairments and multiple co-morbidities, with the likelihood of higher rates of complication after discharge.

Outpatient rehabilitation programs have been successful in reducing symptoms and in improving physi- 
Table 1. Rehabilitation team interventions for cancer patients

\begin{tabular}{|c|c|}
\hline $\begin{array}{l}\text { Physical Medicine and } \\
\text { Rehabilitation Physician } \\
\text { - physiatrist }\end{array}$ & $\begin{array}{l}\text { Inpatient consultation in cancer centre, coordination inpatient and outpatient cancer rehabilitation (prescribes treatments } \\
\text { performed by professionals from other disciplines, such as physical, occupational, and speech therapy et al.), } \\
\text { pharmacologic treatments in pain, spasticity, bowel and bladder dysfunction, mood stabilization, decreased initiation, and } \\
\text { other symptoms and adverse effects, joint injections, trigger point injections, or botulinum toxin injections for symptom } \\
\text { control }\end{array}$ \\
\hline Physical therapist & Strengthening, range of motion exercises, endurance activities, and mobility training (e.g. transfers, gait, stair climbing) \\
\hline Occupational therapist & $\begin{array}{l}\text { Training in activities of daily living such as bathing, grooming, dressing, toileting, meal preparation, and homemaking. In } \\
\text { addition, occupational therapists evaluate home environments for potential modification, provide instruction in driving } \\
\text { with adaptive devices, and implement interventions to promote upper extremity ROM, strength, endurance, and } \\
\text { coordination }\end{array}$ \\
\hline Speech therapist & $\begin{array}{l}\text { Cognitive assessment and training and swallowing evaluation and treatment communication deficits, dysphagia, and train } \\
\text { patients in use of alternative means of speech and communication, including adaptive communication devices, laryngeal } \\
\text { speech, oesophageal speech, and use of a prosthetic larynx. Treatment of patients who have oral defects or experience } \\
\text { aphasia also falls within the purview of the speech therapist }\end{array}$ \\
\hline Psychologist & $\begin{array}{l}\text { Providing assessment and treatment to assist in management of cancer-related psychological distress. As a member of the } \\
\text { rehabilitation team, the psychologist also assists other team members when psychological issues, either in patients or } \\
\text { family members, complicate efforts to provide effective therapy. The goal of consultation of the psychologist with other } \\
\text { team members is to maximize the benefit derived by the patient during the rehabilitation process }\end{array}$ \\
\hline Social worker & $\begin{array}{l}\text { Counselling services to patients and families regarding emotional support, community resources, finances, lifestyle } \\
\text { changes, and treatment participation. In some settings, social workers often serve as leaders for support groups and also } \\
\text { may provide active assistance in discharge planning activities, such as arranging home care services and transfer to other } \\
\text { health care settings }\end{array}$ \\
\hline Dietitian & Teaching patients and family members about the importance of appropriate diet in successful rehabilitation \\
\hline
\end{tabular}

cal and psychosocial functioning for patients during and after oncological treatment. Investigators have also found positive effects of outpatient exercise training, not only in terms of aerobic capacity, strength, and flexibility but also significant gains in multiple domains of quality-of-life (positive affect, decreased distress, enhanced wellbeing, and improved function) [13]. The American College of Sports Medicine (ACSM) recommends rehabilitation programs for patients with cancer diagnosis, on the grounds that these meet the goal of maintaining cardiovascular endurance, muscular strength, and function. The benefits include: decreased nausea, decreased fatigue, increased endurance, and improved quality of life. The 2008 US Department of Health and Human Services (US DHHS) Physical Activity Guidelines for Americans recommends weekly aerobic activity of 150 minutes of moderate intensity exercise or 75 minutes of vigorous-intensity exercise or an equivalent combination. As well as 2-3 weekly sessions of strength training for major muscle groups and stretching of major muscle groups on days which other exercises are performed [7]. The National Comprehensive Cancer Network (NCCN) recommends 30 minutes per day, 5 days per week as a goal for exercise for patients with cancer.

The rehabilitation programs in the United States, Canada, and Germany

The first model of cancer rehabilitation was written in 1940's by dr Howard Rusk with dr Taylor in New Hope for the Handicapped in the United States (US). Cancer as a "special rehabilitation" problem was described in the chapter on rehabilitation of surgical patients [14]. First volume by Howard Rusk Rehabilitation Medicine contained a full chapter on cancer rehabilitation in the initial 1958 edition [15].

The next stage of development of this program took place in 1971 when the National Cancer Act was passed, and funds became more readily available for the development of training, demonstration, and research projects in rehabilitation and were administered through the Division of Cancer Control and Rehabilitation, National Cancer Institute $(\mathrm{NCl})$ of the National Institute of Health (NIH) [16, 17].

The cancer rehabilitation history certainly would not be complete without the pioneer rehabilitation programs. Two early programs were conducted at the University of Texas MD Anderson Cancer Center and in New York [18].

At present, at MD Anderson Cancer Center in Texas, in the US, the inpatient cancer rehabilitation interdisciplinary team includes a physiatrist, a nurse practitioner, a physical therapist, an occupational therapist, a speech therapist, a rehabilitation nursing specialist, a nutritionist, a pharmacist, a case manager, working together to achieve the goal of safe patient discharge [19]. This team works closely with primary medical and surgical oncologists to coordinate care for ongoing chemotherapy, complex surgical wounds, adverse effects 
from treatment, and the effects of disease progression. Ready access to medical and surgical specialists, internists, and intensive care specialists is required to manage the complex acute issues which these patients can present. An exception to this rule is a subset of patients who have developed significant deconditioning after systemic chemotherapy, and the rehabilitation goal is to improve their function back up to the performance level at which they would be eligible for their next round of systemic chemotherapy. These patients' planned discharge disposition is to go back to their oncology team for further inpatient treatment [20, 21, 22].

In Canada, in 1997, the Ottawa Regional Cancer Centre began to offer an Oncology Rehabilitation Program to patients with cancer. The primary goal of this program was to improve the quality of life, functional performance and psychosocial adjustment of patients with cancer who were undergoing active therapy. An indoor walking and jogging track, a group exercise area, free weights and an array of strength and aerobic training machines are available [23]. If the patient is eligible for participation, an exercise specialist (an exercise physiologist with a relevant degree) completes a compulsory physical fitness assessment and an optional nutritional assessment and determines baseline values for health-related quality of life.

In Germany, in turn, rehabilitation is an integral part of a comprehensive social security system which roots date back to the $19^{\text {th }}$ century. The slogan "rehabilitation before retirement" captures the idea of rehabilitation as a prevention of early retirement in a nutshell. Based on the historical background of the German social security system, the German rehabilitation system evolved as a specific and independent system which is unique and distinct from the system in many other European countries where rehabilitation measures are part of primary health care [24]. Nowadays, based on the social laws, rehabilitation measures are mainly carried out as inpatient programs in specialized rehabilitation clinics, which are staffed with multidisciplinary rehabilitation teams.

\section{The drawback issues of cancer rehabilitation}

More rehabilitation professionals (including physiatrists - physical medicine and rehabilitation physican, physical therapists, occupational therapists, and speech and language pathologists, social workers) need to have specific training in the field of oncology rehabilitation. The American Academy of Physical Medicine and Rehabilitation has developed a Medical Rehabilitation Council that specifically contains a Cancer Rehabilitation Medical Sub- specialty Group to help physiatrists share information, network, and foster the development of oncology rehabilitation [25]. Oncologists and other physicians need education on appropriate screening for and referral to rehabilitation for cancer patients. This cross-disciplinary interaction is an important part of developing and fostering oncology rehabilitation services. Interdisciplinary care for oncology patients and cancer survivors needs to become part of the standard of treatment. Rehabilitation and oncology professionals need to find ways to work together to provide optimal cancer rehabilitation services to the many patients who need them. There is clearly a significant deficit in cancer care when rehabilitation is not offered to those who will likely benefit from it. Bridging the gap between these two disciplines can be challenging but is an important goal to provide the best possible care for cancer survivors. By improving physical functioning, we can also positively influence the survivor's social and emotional functioning [26]. In some cases, a patient-centred approach with an individualized comprehensive treatment plan may need to be developed for the survivor. This is best accomplished by means of an interdisciplinary rehabilitation team that also includes expertise in, for example, occupational therapy, nutrition, speech-language pathology and exercise physiology.

\section{Weaknesses of the study}

The paper does not take into account the poor accessibility and financing the cancer rehabilitation services of the holistic approach in cancer care despite their obvious benefits.

\section{Conclusion}

Cancer rehabilitation can occur in various settings, depending on the level of disability, extent of disease, medical acuity level of the patient, and available services. Rehabilitation has substantial effects on the patients' physical, psychological, social and existential well-being. There is strong evidence that rehabilitation is a well-tolerated and safe adjunct therapy that can mitigate several common treatment-related side effects among cancer patients.

\section{Conflict interest}

The authors indicated no potential conflicts of interest.

\section{References}

1. Holm LV, Hansen DG, Johansen C, Vedsted P, Larsen PV, Kragstrup J, et al. Participation in cancer rehabilitation 
and unmet needs: a population-based cohort study. Support Care Cancer. 2012;20:2913-24.

2. World Health Organisation. ICF: International Classification of Functioning and Disability and Health. 2001; http://www3.who.int/icf/icftemplate.cfm.

3. Stucki G, Melvin J. The International Classification of Functioning, Disability and Health: A unifying model for the conceptual description of physical and rehabilitation medicine. J Rehabil Med. 2007;39:286-92.

4. White book on Physical and Rehabilitation Medicine in Europe. J Rehabil Med. 2007;45(Suppl):6-47.

5. Disler PB, Cameron ID, Wilson SF. Rehabilitation medicine. Med J Aust. 2002;177:385-6.

6. Thorsen L, Gjerset GM, Loge JH, Kiserud CE, Skovlund E, Fløtten $T$, et al. Cancer patients' needs for rehabilitation services. Acta Oncol. 2011;50:212-22.

7. Schmitz KH, Courneya KS, Matthews C, Demark-Wahnefried W, Galvão DA, Pinto BM, et al. American College of Sports Medicine roundtable on exercise guidelines for cancer survivors. Med Sci Sports Exerc. 2010;42: 1409-26.

8. Dimeo F, Rumberger BG, Keul J. Aerobic exercise as therapy for cancer fatigue. Clin Sci. 1998;30:475-8.

9. Speck RM, Courneya KS, Masse LC, Duval S, Schmitz KH. An update of controlled physical activity trials in cancer survivors: A systematic review and meta-analysis. J Cancer Surviv. 2010;4:87-100.

10. Tay SS, Ng YS, Lim PA. Functional outcomes of cancer patients in an inpatient rehabilitation setting. Ann Acad Med Singapore. 2009;38:197-201.

11. DePompolo RW. Development and administration of a cancer rehabilitation program. Phys Med Rehabil State Art Rev. 1994;8:413-23.

12. Yoshioka H. Rehabilitation for the terminal cancer patient. Am J Phys Med Rehabil. 1994;73:199-206.

13. Silver JK, Gilchrist LS. Cancer Rehabilitation with a Focus on Evidence-Based Outpatient Physical and Occupational Therapy Interventions Am J Phys Med Rehabil. 2011;90:5-15.

14. Rusk HA, Taylor EJ. New Hope for the Handicapped. Harper and Brothers, New York 1946.

15. Rusk HA. Rehabilitation Medicine. CV Mosley, St. Louis 1958.

16. Harrey RF, Jellinek HM, Habeck RV. Cancer rehabilitation: An analysis of the 36 program approaches. JAMA. 1982;247:2127-31.
17. Mayer DK. The healthcare implications of cancer rehabilitation in the twenty-first country. Oncol Nurs Forum. 1992;19:23-7.

18. Dietz JH. Introduction. In: Dietz JH. Rehabilitation Oncology. John Wiley\&Sons, New York 1981, 1-2.

19. Guo Y, Palmer JL, Kaur G, Hainley S, Young B, Bruera E. Nutritional status of cancer patients and its relationship to function in an inpatient rehabilitation setting. Support Care Cancer. 2005;13:169-75.

20. Sagen A, Karesen R, Risberg MA. Physical activity for the affected limb and arm lymphedema after breast cancer surgery. A prospective, randomized controlled trial with two years follow-up. Acta Oncol. 2009;48:1102-10.

21. Jorgensen IL, Frederiksen K, Boesen E, Elsass P, Johansen C. An exploratory study of associations between illness perceptions and adjustment and changes after psychosocial rehabilitation in survivors of breast cancer. Acta Oncol. 2009;48:1119-27.

22. Shin KY, Guo Y, Konzen B, Fu J, Yadav R, Bruera E. Inpatient cancer rehabilitation: The Experience of a National Comprehensive Cancer Center. Am J Phys Med Rehabil. 2011;90:63-8.

23. Segal R, Evans W, Johnson D, Smith J, Colletta SP, Corsini $\mathrm{L}$, et al. Oncology Rehabilitation Program at the Ottawa Regional Cancer Centre: program description. CMAJ. 1999;161:282-5.

24. Hohmann J. Gesundheits-, Sozial- und Rehabilitations systeme in Europa. Hans Huber, Bern 1998.

25. Institute of Medicine. From Cancer patient to cancer survivor: Lost in transition. National Academies Press, Washington 2005.

26. Gamble GL, Gerber LH, Spill GR, Paul KL. The future of cancer rehabilitation: emerging subspecially. Am J Phys Med Rehabil. 2011;90:76-87.

\section{Correspondence address:} Katarzyna Hojan Department of Rehabilitation the Greater Poland Cancer Centre 15 Garbary Street, 61-866 Poznań, Poland phone: +48618850705

fax: +48 618521948 email: khojan@op.pl 